\title{
PROPULSIÓN Y DIRECCIÓN DE UN MICRORROBOT TIPO FLAGELO FLEXIBLE UTILIZANDO LA ANALOGÍA DEL COHETE. SIMULACIÓN EN COMSOL MULTIPHYSICS
}

\author{
E. Mancha-Sánchez*, P. Rodríguez, A. Bravo, I. Tejado, B.M. Vinagre* \\ \{emancha*,palomard,abravoi,itejbal,bvinagre*\}@unex.es
}

Escuela de Ingenierías Industriales, Universidad de Extremadura, 06006 Badajoz

Resumen

Los microrrobots tienen el potencial de revolucionar la medicina. Por el momento se encuentran muchas limitaciones, entre las que destacan la fabricación, el suministro de energía y la propulsión. En este trabajo se utiliza la simulación computacional para realizar un estudio sobre la propulsión de un microrrobot tipo flagelo flexible teniendo en cuenta estas limitaciones haciendo una analogía con la propulsión de un cohete. Utilizando COMSOL Multiphysics se ha simulado un microrrobot autónomo con flagelo flexible impulsado por reacciones químicas, que han sido emuladas con la aplicación de distintas fuerzas aplicadas en la cabeza. Los resultados obtenidos permiten concluir que el microrrobot es propulsado tanto por las fuerzas aplicadas, como por el movimiento generado por el flagelo. Además, el flagelo realiza una función de estabilización del microrrobot.

Palabras clave: Microrrobot, Autónomo, Flagelo, Simulación computacional, Cohete.

\section{INTRODUCCIÓN}

La robótica se ha convertido en una de las disciplinas más prometedores para la medicina. Desde la cirugía robótica a los microrrobots. Estos últimos, dado su tamaño, pueden permitir una cirugía mínimamente invasiva con aún menos complicaciones [1], la liberación de fármacos de manera dirigida y controlada [2] o su utilización como biosensores [3]. Estos microrrobots están gobernados por distintas físicas que en el mundo macroscópico debido a su pequeño tamaño. En este aspecto, el número de Reynolds, es decir la relación entre las fuerzas inerciales y las fuerzas viscosas, se reduce ostensiblemente, de tal manera que las fuerzas viscosas se convierten en las dominantes, haciendo insignificante las fuerzas inerciales. Los sistemas de propulsión de estos microrrobots tienen que tener en cuenta estos factores.

La mayor parte de los mecanismos de propulsión suelen fijarse en los sistemas biológicos, ya sean macroscópicos, como los peces [4], o microscópi- cos, como los flagelos bacterianos o los espermatozoides [5]. Del mismo modo, se utilizan sistemas de propulsión basados en la producción de propelentes que impulsen el microrrobot, generalmente mediante reacciones químicas, producidas por un catalizador situado en el microrrobot mediante compuestos presentes en el medio [6].

La fuente de energía sigue siendo uno de los elementos limitantes en el desarrollo de un microrrobot. Existen multitud de aproximaciones a la hora de alimentar al microrrobot: microrrobots impulsados por un campo magnético que le permiten mover un flagelo [7], activados por radiación infrarroja [8], mediante ultrasonidos [9], o a través de un campo eléctrico que hace girar una hélice [10]. Este tipo de microrrobots se denominan dirigidos o no autónomos, ya que necesitan de una fuente de energía externa a él para su movimiento. Presentan ciertas ventajas frente a los autónomos, en cuanto a que se puede modificar de manera más sencilla la energía aportada, permitiendo modular la cantidad de movimiento que genera el microrrobot. Por otro lado, aquellos microrrobots que poseen un sistema de propulsión, sin la necesidad de intervención directa, se conocen como autónomos. La energía de estos microrrobots suele provenir del entorno, por reacciones químicas, gradientes de $\mathrm{pH}$, de temperatura, etc. La ventaja de este tipo de microrrobots es que permite una navegación autónoma por el organismo [11].

La dificultad de fabricación, control y suministro de energía de estos microrrobots pone en valor la utilización de herramientas alternativas a las tradicionales a la hora de hacer una primera aproximación al estudio de los microrrobots, así como para su optimización. La simulación computacional destaca a la hora de realizar este trabajo. Existen multitud de ejemplos en los que esta herramienta se ha utilizado anteriormente. Estos trabajos previos se basan tanto en la simulación de las condiciones en las que el microrrobot se sumerge, como en la actuación, o la forma de propulsión $[12,13,14]$.

El objetivo de este trabajo es realizar una simulación computacional de un microrrobot autónomo propulsado por una reacción química. Esta reac- 
XLII Jornadas de Automática

ción, situada en la cabeza del microrrobot, genera par de rotación en la cabeza que se desplaza hasta el flagelo, provocando éste un desplazamiento del microrrobot. Se estudiaron los desplazamientos del microrrobot con y sin flagelo para demostrar la necesidad del flagelo. Además se estudiaron las velocidades y aceleraciones producidas como consecuencia de las fuerzas aplicadas.

\section{MATERIALES Y MÉTODOS}

\subsection{MODELO}

El modelo está compuesto por un microrrobot formado por una cabeza tipo elipsoide y un flagelo basado en [15], así como un canal, en el que el microrrobot fluye, con las medidas especificadas en la Tabla 1. Para las simulaciones se utilizó COMSOL Multiphysics con un modelo en 2D aplicando una interacción Fluido-Estructura (FSI, por sus siglas en inglés), en la que tanto el microrrobot (dominio sólido) como el fluido interaccionan mutuamente.

Tabla 1: Medidas de los elementos del modelo

\begin{tabular}{|c|c|}
\hline Elemento & Tamaño (ancho $\mathbf{x}$ alto) $\mathbf{( m m )}$ \\
\hline Canal & $9 \times 25-50$ \\
Cabeza & Semieje a 0,342 \\
& Semieje b 0,529 \\
Flagelo & $0,064 \times 4$ \\
\hline
\end{tabular}

Para esta simulación, se utilizó como fluido agua y, para los sólidos, politetrafluoroetileno (PTFE) para la cabeza y polidimetilsiloxano (PDMS) para el flagelo. Se realizaron distintos estudios sobre el movimiento y desplazamiento producido, tanto únicamente en la cabeza como en el microrrobot completo, al aplicarle una fuerza equivalente a la producida con la reacción química de impulso deseada. Para ello se utilizaron dos configuraciones distintas del modelo. En el primero (modelo 1) sólo se incluyó la cabeza sobre el que se le aplicaron las fuerzas dentro del canal con el fluido. El segundo (modelo 2) incluía el microrrobot completo (cabeza y flagelo) en el canal. Las fuerzas que genera la reacción química se aproximaron mediante la aplicación de fuerzas alternas a los lados de la cabeza como se detalla en el apartado 2.3.

\subsection{ECUACIONES DINÁMICAS}

Para el dominio fluídico, de acuerdo con un fluido Newtoniano incompresible, las ecuaciones de continuidad y momento de Navier-Stokes son:

$$
\rho \nabla \cdot u_{\text {fluid }}=0
$$

Modelado, Simulación y Optimización

$$
\begin{array}{r}
\rho \frac{\partial u_{\text {fluid }}}{\partial t}+\rho\left(u_{\text {fluid }} \cdot \nabla\right) u_{\text {fluid }}= \\
\nabla \cdot\left[-p I+\mu\left(\nabla u_{\text {fluid }}+\nabla\left(u_{\text {fluid }}\right)^{T}\right)\right]+F
\end{array}
$$

donde $\rho$ es la densidad del fluido, $u_{\text {fluid }} \mathrm{su}$ velocidad, $p$ la presión, $I$ la matriz identidad, $\mu$ la viscosidad dinámica, $T$ la temperatura $\mathrm{y}$ $F$ la fuerza externa aplicada al fluido. En esta simulación las fuerzas externas provienen de las fuerzas aplicadas en la cabeza, que interactúan con el dominio fluido debido a las físicas acopladas del FSI.

Para el dominio sólido, la ecuación de equilibrio viene dada por:

$$
\rho \frac{\partial^{2} u_{\text {solid }}}{\partial t^{2}}-\nabla \cdot \sigma=F_{V}
$$

donde $\sigma$ es el tensor tensión de Cauchy, $F_{V}$ la fuerza del cuerpo y $u_{\text {solid }}$ la deformación del sólido. El tensor tensión de Cauchy se calcula del segundo tensor tensión de Piola-Kirchhoff $S$ con:

$$
\rho=J^{-1} F S F^{T}
$$

donde $J$ es el Jacobiano de la deformación, dado por:

$$
J=|F|
$$

y $F$, el gradiente de deformación, se puede expresar en términos del gradiente del vector desplazamiento $u_{\text {solid }}$ como:

$$
F=I+\nabla u_{\text {solid }}
$$

El tensor de deformación del material elástico lineal $\epsilon$ está escrito en términos del gradiente de desplazamiento:

$\epsilon=\frac{1}{2}\left[\left(\nabla u_{\text {solid }}\right)^{T}+\nabla u_{\text {solid }}+\left(u_{\text {solid }}\right)^{T} u_{\text {solid }}\right]$

La ley de Hooke relaciona el tensor de tensión, el tensor de deformación y la temperatura como sigue:

$$
s=s_{0}+c:\left(\epsilon-\epsilon_{0}-\epsilon_{t h}\right)
$$

donde $c$ es el $4^{\circ}$ orden del tensor elástico, el operador ":" es el producto escalar de los dos tensores, $s_{0}$ y $\epsilon_{0}$ son las tensiones y deformaciones iniciales, $\epsilon_{t h}=\alpha\left(T-T_{r e f}\right)$ es la dilatación térmica y $\alpha$ es el coeficiente de expansión térmica.

Para el FSI, se utiliza el método LagrangianoEuleriano arbitrario (ALE) [16] para resolver los dominios sólidos y fluidos. La fuerza total ejercida en el contorno sólido por parte del fluido es:

$$
f_{r}=n \cdot\left[-p I+\mu\left(\nabla u_{\text {fluid }}+\left(\nabla u_{\text {fluid }}\right)^{T}\right)\right]
$$


XLII Jornadas de Automática

donde, $n$ es la normal. La fuerza aplicada en el controno del dominio sólido está dada:

$$
F_{V}=\rho \frac{\partial^{2} u_{\text {solid }}}{\partial t^{2}}-\nabla \cdot \sigma
$$

donde $F_{V}$ es la fuerza volumétrica que está calculada por:

$$
F_{V}=\frac{F_{t o t}}{V}
$$

donde $F_{\text {tot }}$ es la fuerza aplicada y $V$ el volumen del dominio sólido. Una transformación de la fuerza es necesaria para acoplar el marco espacial (ecuaciones de Navier-Stokes) y el material (interfaz de mecánica de sólidos), esto se hace de acuerdo a:

$$
F=f \frac{d v}{d V}
$$

donde $d v$ y $d V$ son los factores de escala de los elementos de malla para el marco espacial y para el de material, respectivamente. El acoplamiento en la otra dirección consiste en la velocidad de la estructura $\frac{\delta u_{\text {solid }}}{\delta t}$ que actúa como una pared móvil para el dominio fluido.

\subsection{DOMINIOS Y CONDICIONES DE CONTORNO}

Para el dominio fluídico se utilizó agua como material, con una densidad de $1000 \mathrm{~kg} / \mathrm{m}^{3}$ y una viscosidad dinámica de 0,001 $\mathrm{Pa} \cdot \mathrm{s}$.

Para el dominio sólido se usó PDMS para el flagelo, con una densidad de $970 \mathrm{~kg} / \mathrm{m}^{3}$, un módulo de Young de $750 \mathrm{kPa}$ y un coeficiente de Poisson de 0,49. Para el material de la cabeza se usó PTFE con una densidad es de $2200 \mathrm{~kg} / \mathrm{m}^{3}$, un módulo de Young de $400 \mathrm{MPa}$ y un coeficiente de Poisson de 0,46.

Con el modelo 1, el microrrobot sin el flagelo, se realizaron dos simulaciones distintas. En la primera se aplicó un impulso de $0,05 \mathrm{mN}$ en un punto de la cabeza (Figura 1a) a $45^{\circ}$ del sistema de referencia global. En la segunda se aplicaron unas fuerzas alternas de $0,05 \mathrm{mN}$ de periodo 0,025 s en dos contornos de la cabeza (Figura 1b). Para las fuerzas aplicadas, cuando el periodo de actuación de una fuerza terminaba, comenzaba el siguiente. Cada una de las fuerzas se mantiene de manera independiente un tiempo de actuación de un $10 \%$ del periodo total, siendo este, en consecuencia de 0,25 s (Figura 3).

En el modelo 2, el microrrobot con el flagelo, se realizaron seis simulaciones distintas. En la primera de ellas se aplicó un impulso en un punto de la cabeza del microrrobot del mismo modo que
Modelado, Simulación y Optimización

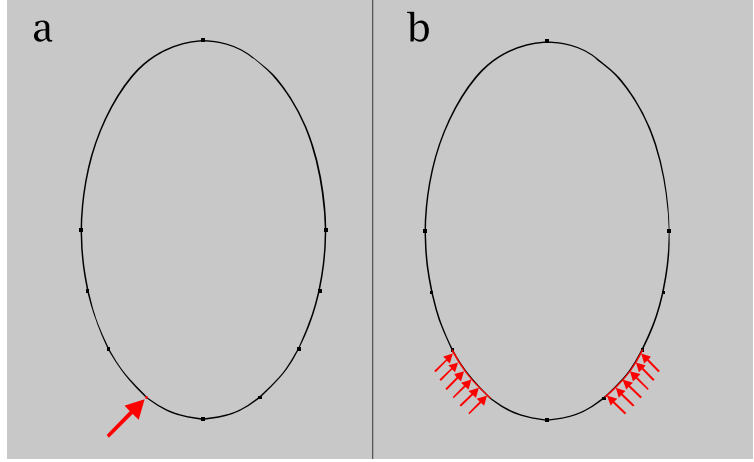

Figura 1: Localización de las fuerzas en microrrobot con sólo la cabeza. La flecha roja indica el lugar donde se aplica la fuerza y su sentido. a) Impulso puntual, b) pulso de fuerzas alternas aplicadas en los contornos marcados, primero se aplica el de la izquierda e inmediatamente después el de la derecha.

la primera simulación del primer modelo (Figura 2a). En este caso, debido a la presencia del flagelo, es necesario aplicar una fuerza mayor para desplazar el microrrobot alcanzando los 0,5 mN. En las otras cinco simulaciones se aplicaron dos fuerzas alternas en la cabeza del microrrobot (Figura 2b). El periodo de activación de las fueras se mantuvo $(0,025 \mathrm{~s})$, reduciéndose el periodo de la onda progresivamente. Pasando el periodo de activación de un $10 \%$ del periodo al $30 \%$ con pasos de $5 \%$, esto es 10, 15, 20, 25, $30 \%$ (Figura 3).

\subsection{MALLADO Y SIMULACIÓN}

Se creó una malla 2D con elementos cuadrangulares y triangulares (Figura 4). Se utilizaron elementos cuadrangulares para los contornos de las geometrías. Los elementos triangulares se utilizaron para el resto de las geometrías no malladas. Los elementos cuadrangulares y triangulares generados en el microrrobot fueron de 224 y 484 , respectivamente. Para el canal, como su tamaño variaba en longitud, se generaron más o menos elementos en las distintas configuraciones siendo el máximo número de elementos 898 y 12634, para los elementos cuadrangulares y triangulares, respectivamente. Para las simulaciones sobre las que se preveía un mayor desplazamiento del microrrobot se utilizó una regeneración de malla automática mediante el atributo de COMSOL "Remallado automático". Con este atributo se conseguía que la malla se regenerara al alcanzar un valor máximo dado de distorsión. El tiempo de simulación fue de $3 \mathrm{~s}$. Se usó un resolvedor totalmente acoplado que utiliza MUMPS como resolvedor lineal y un método no lineal con un factor de amortiguamiento automático. 


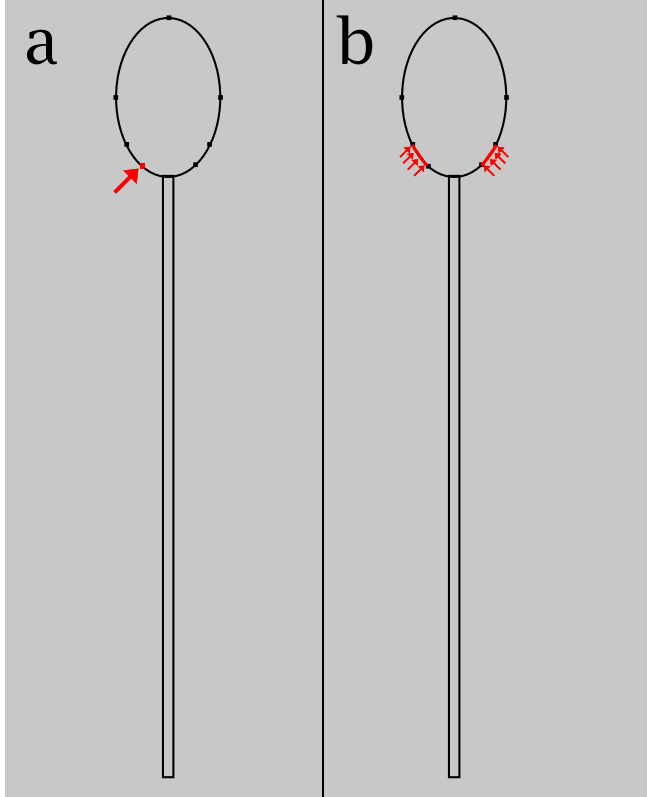

Figura 2: Localización del pulso de fuerza aplicado al microrrobot con flagelo. La flecha roja indica el lugar donde se aplica la fuerza y su sentido. a) Impulso puntual, b) pulso de fuerzas alternas aplicadas en los contornos marcados, primero se aplica el de la izquierda e inmediatamente después el de la derecha.

\section{RESULTADOS Y DISCUSIÓN}

\subsection{DESPLAZAMIENTO}

Para cada uno de los experimentos realizados con cada modelo se estudió el desplazamiento que la cabeza realizaba en los ejes $x$ e $y$. En el modelo 1 la aplicación de un impulso puntual de la fuerza genera una deriva lateral (Figura 5). Por otro lado, la aplicación de las fuerzas alternas anteriormente descritas, genera un desplazamiento principalmente a lo largo del eje y. Como se observa en la Figura 5 la aplicación de estas fuerzas, también genera un desplazamiento no deseado a lo largo del eje x que propicia que la cabeza adquiera un desplazamiento descontrolado.

En el modelo 2, el que posee flagelo, el desplazamiento provocado por la aplicación del impulso puntual (Figura 6) sigue una tendencia muy similar al modelo sin flagelo. En este caso, se provoca un desplazamiento similar tanto en $x$ como en $y$. En aquellos microrrobots a los que se les aplicaron fuerzas alternas, presentan un desplazamiento mayoritariamente en el eje $y$. Como se observa en la Figura 6, conforme aumenta el porcentaje de actuación de las fuerzas sobre la cabeza del microrrobot, aumenta el desplazamiento provocado. El mayor desplazamiento se produce con la aplica-

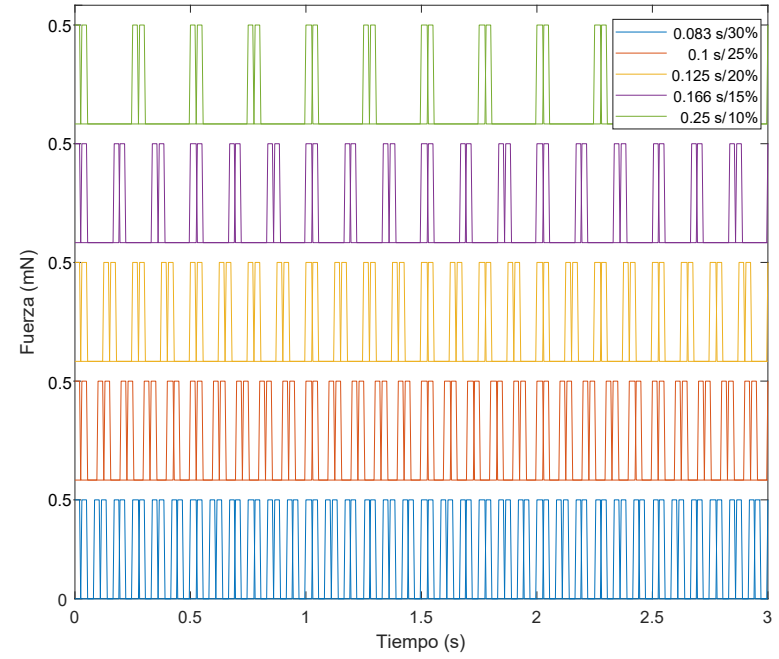

Figura 3: Gráfico de fuerzas aplicadas a la cabeza y el microrrobot.

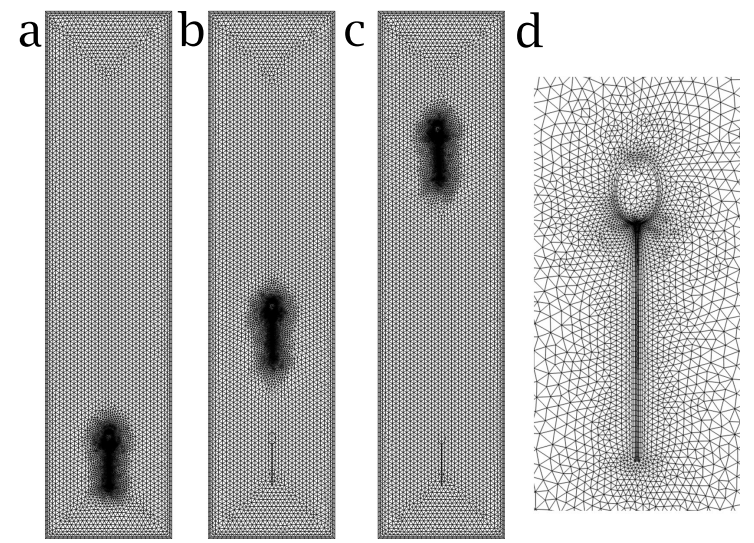

Figura 4: Mallado del microrrobot con flagelo. Remallado a distintos tiempos a) $0 \mathrm{~s}$, b) $1,5 \mathrm{~s}$, c) $3 \mathrm{~s}$. d) Detalle del mallado en la zona del microrrobot.

ción de la fuerza con mayor porcentaje de actuación, en este caso $30 \%$ con 29,24 mm. En la Tabla 2 se muestran los desplazamientos máximos de cada microrrobot. En la gráfica se observa además, que apenas existe desplazamiento en el eje $x$. Sin embargo, como se observa la Figura 7 este desplazamiento en $x$ no es cero, sino que debido a la presencia alterna de las fuerzas aplicadas se produce un movimiento ondulatorio. A pesar de esto, el desplazamiento en $x$ es ostensiblemente menor en el microrrobot con flagelo que sin él, además teniendo en cuenta que la fuerza aplicada en el microrrobot con flagelo es 10 veces mayor. Es decir, la presencia del flagelo favorece la estabilización del movimiento.

La estabilización provocada por el flagelo puede ser asimilada a la acción del combustible en un 


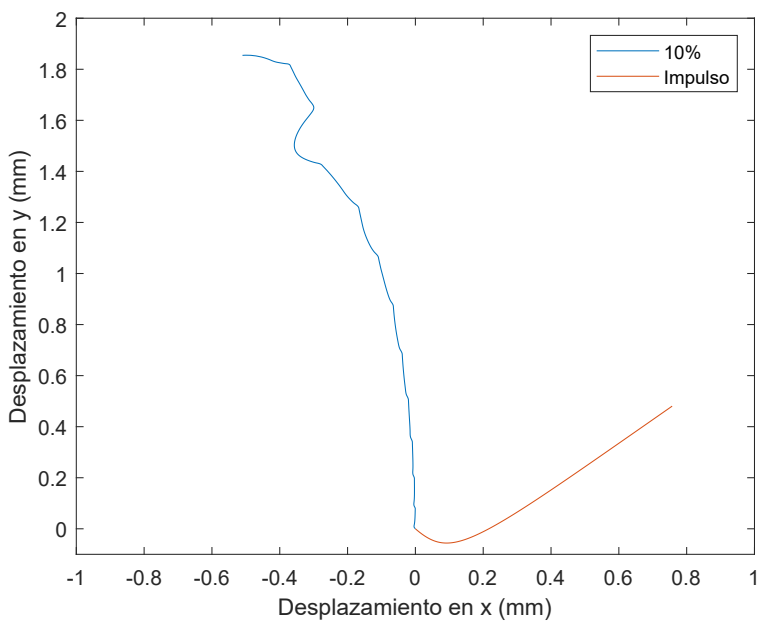

Figura 5: Desplazamiento de la cabeza en $x$ e $y$ con la aplicación de un par de fuerzas (10\%) y con un impulso puntual (Impulso).

cohete como se modela en [17] (Figura 8a). En él, la acción del combustible se asemeja a la de un péndulo que se desplaza de manera dependiente a la rotación del cohete debido a la acción de sus propulsores. Estos propulsores, situados lateralmente y de manera trasera, estabilizan e impulsan, respectivamente, al cohete. En nuestro caso, la cabeza del microrrobot sería el cohete y los propulsores, tanto los laterales (componente $x$ de las fuerzas aplicadas) como el trasero (componente $y$ de las fuerzas). Por su parte, el flagelo actuaría tanto de propulsor como de péndulo, en este caso situado fuera del cuerpo. Como se observa en la Figura 8b, el flagelo, por cada componente $x$ de la fuerza, genera una reacción en dirección contraria, haciendo que el sistema se estabilice (péndulo). Con este movimiento, además de estabilizar el microrrobot, se genera una forma de onda que lo impulsa haciendo las veces del propulsor del cohete.

\subsection{VELOCIDAD}

Se estudió la velocidad alcanzada por los microrrobots con flagelo. En la Figura 9 se observan las velocidades alcanzadas por los cinco microrrobots con flagelo. Como se ve en la figura, todas las curvas de velocidad presentan el mismo patrón. Utilizando como ejemplo la curva de $10 \%$, se observa cómo se produce un aumento brusco por cada par de fuerzas aplicadas. Observando detalladamente cada uno de esos picos, se ve cómo cada aumento presenta un primer pico, correspondiente a la primera fuerza aplicada, e inmediatamente después otro con la siguiente fuerza del par. Esta aplicación consecutiva de fuerzas permite que el aumento de velocidad se

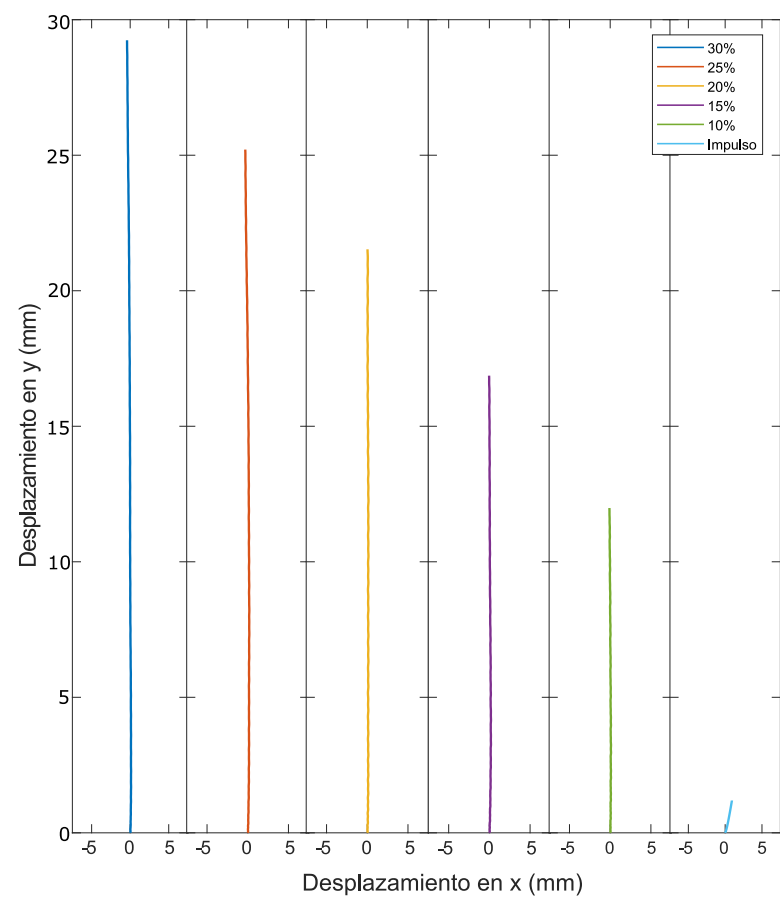

Figura 6: Desplazamiento de los microrobots con flagelo en $x$ e $y$.

vaya acumulando, siendo esta acumulación mayor en aquel modelo en el que las fuerzas se dan con mayor frecuencia $(30 \%)$. Estos cambios en la velocidad son una relación directa a la aceleración provocada por las fuerzas aplicadas, como se comenta en el apartado 3.3. Esta velocidad termina estabilizándose a lo largo del tiempo. La velocidad media estabilizada, así como la velocidad máxima alcanzada pueden verse en la Tabla 2 .

\subsection{ACELERACIÓN}

Por otro lado la aceleración producida en el movimiento es un parámetro importante a tener en cuenta para comprobar si el movimiento se está produciendo únicamente por el impulso generado por la fuerza o si, a su vez, este impulso y movimiento de la cabeza hace que el flagelo se mueva y genere su propio impulso. En la Figura 10 se observan las aceleraciones producidas en cada uno de los microrrobots con flagelo. Se puede ver cómo existen unos picos muy pronunciados de aceleración repetitivos provocados por las fuerzas externas aplicadas. Se observa cómo la aceleración no cae a cero como cabría esperar al eliminar las fuerzas externas. De este modo, una vez decae el impulso generado por las fuerzas aplicadas, se puede observar una serie de aceleraciones de menor 
Tabla 2: Desplazamiento, velocidad media en estacionario y velocidad máxima de cada uno de los microrrobots

\begin{tabular}{|c|c|c|c|}
\hline Fuerzas & $\begin{array}{c}\text { Desplazamiento } \\
\text { máximo }(\mathbf{m m})\end{array}$ & $\begin{array}{c}\text { Velocidad media } \\
\text { estacionario } \mathbf{( m m} / \mathbf{s})\end{array}$ & $\begin{array}{c}\text { Velocidad } \\
\text { máxima }(\mathbf{m m} / \mathbf{s})\end{array}$ \\
\hline $10 \%$ & 11,98 & 4,87 & 7,08 \\
$15 \%$ & 16,86 & 6,80 & 8,79 \\
$20 \%$ & 21,52 & 8,55 & 10,41 \\
$25 \%$ & 25,20 & 9,82 & 11,16 \\
$30 \%$ & 29,24 & 11,31 & 12,43 \\
\hline
\end{tabular}

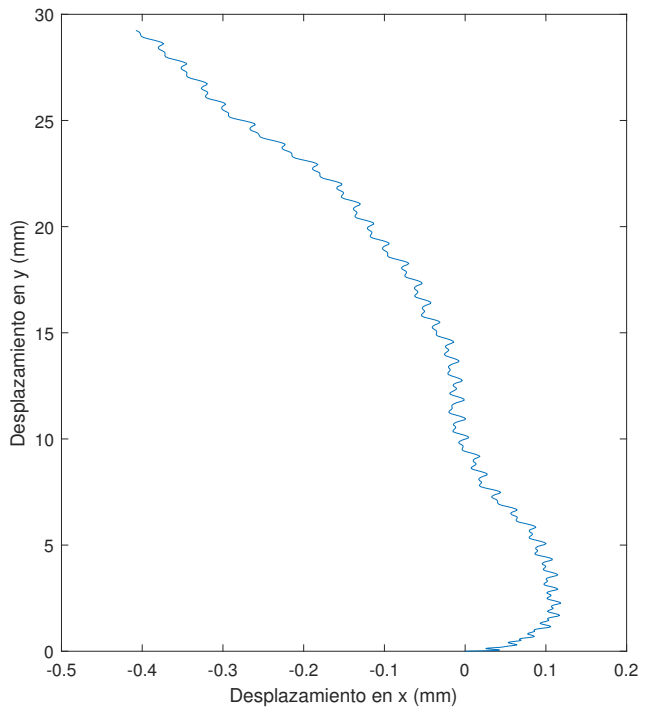

Figura 7: Desplazamiento del microrrobot con un $30 \%$ de fuerza aplicada en $x$ e $y$. Nótese la escala de eje $x$.

intensidad entre cada grupo de picos de aceleración. Estas aceleraciones más débiles son las que se producen debido al movimiento ondulatorio del flagelo, provocado, a su vez, por el par generado por las fuerzas aplicadas en la cabeza.

\section{CONCLUSIONES}

En este trabajo se han llevado a cabo una serie de simulaciones computacionales de un microrrobot autónomo con flagelo flexible. En ellas, se realizó un primer estudio sobre la cinemática de la cabeza del microrrobot para conocer el efecto del flagelo, mostrando que, sin el flagelo, el movimiento provocado por el par de fuerzas tenía una pobre estabilidad relativa. Por otro lado, se aplicaron fuerzas alternas al microrrobot con flagelo para observar su movimiento y el valor añadido que aporta el flagelo a su movimiento. El flagelo en el microrrobot genera una propulsión añadida a las fuerzas aplicadas debido al movimiento que generan éstas en la cabeza. Por lo tanto

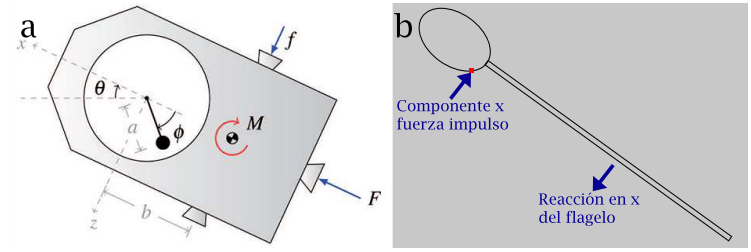

Figura 8: Similitudes entre: a) el cohete modelado por [17] y b) nuestro microrrobot.

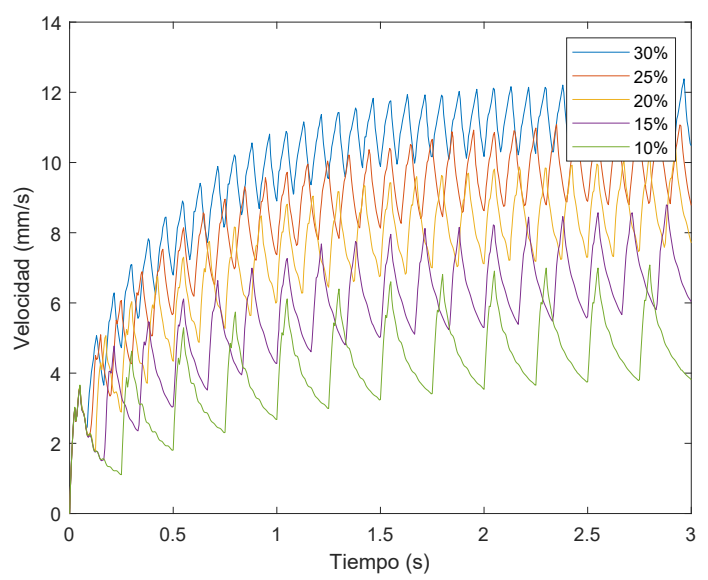

Figura 9: Velocidad de cada microrrobot con flagelo.

se ha demostrado la utilidad del flagelo como sistema estabilizador, así como para favorecer la propulsión.

Para poder controlar la dirección y la velocidad de movimiento son necesarias más simulaciones en las que se estudie las fuerzas de sustentación y el arrastre generado por el flagelo, así como la forma de onda generada. Además, es necesaria una optimización de los parámetros de entrada de la simulación, como son la fuerza aplicada, su dirección, y los materiales utilizados para la cabeza y el flagelo. El siguiente paso para poder comprender cómo funciona este microrrobot es realizar un modelo matemático asemejándolo a la propulsión de un cohete. 


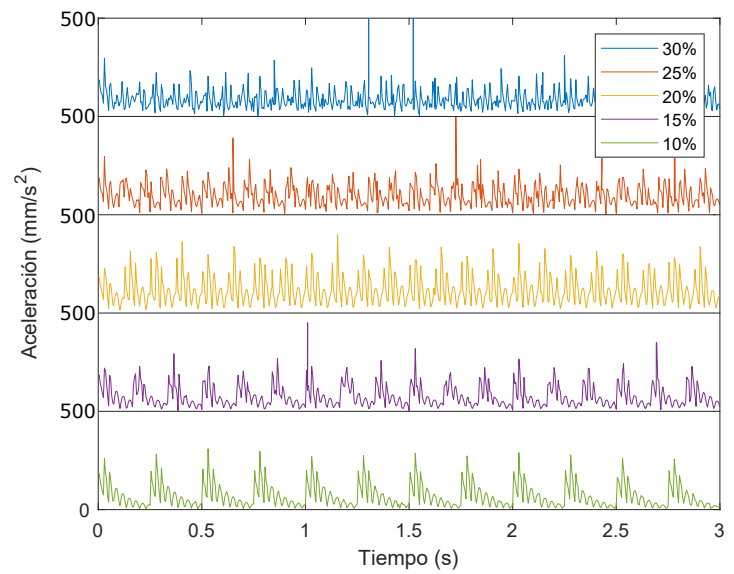

Figura 10: Aceleración de cada microrrobot con flagelo.

\section{Agradecimientos}

Este artículo ha sido financiado por la Consejería de Economía, Ciencia y Agenda Digital de la Junta de Extremadura mediante el proyecto IB18109 y la "Ayuda a Grupos" GR18159, por la Agencia Estatal de Investigación mediante el proyecto PID2019-111278RB-C22 / AEI / $10.13039 / 501100011033$, y por los Fondos Europeos de Desarrollo Regional "Una manera de hacer Europa".

\section{English summary}

\section{PROPELLING AND STEERING OF A FLEXIBLE FLAGELLUM- LIKE MICROROBOT USING ROC- KET ANALOGY. SIMULATION IN COMSOL MULTIPHYSICS}

\author{
Abstract \\ Microrobots have the potential to revolutio- \\ nize medicine. At the moment, there are \\ many limitations, among which the manu- \\ facturing, the power supply and the pro- \\ pulsion stand out. In this work, compu- \\ tational simulation is used to carry out a \\ study on propulsion of a microrobot with \\ flexible flagellum taking into account the- \\ se limitations, making an analogy with the \\ propulsion of a rocket. Using COMSOL \\ Multiphysics, an autonomous microrobot \\ with a flexible flagellum driven by chemical \\ reactions has been simulated. These reac- \\ tions have been emulated with the applica-
}

tion of different forces applied to the head. The results obtained allow us to conclude that the micro-robot is propelled both by the applied forces and by the movement generated by the flagellum. In addition, the flagellum performs a stabilization function of the microrobot.

Keywords: Microrobot, Autonomous, Flagellum, Computational Simulation, Rocket.

\section{Referencias}

[1] B. J. Nelson, I. K. Kaliakatsos, and J. J. Abbott, "Microrobots for minimally invasive medicine," Annual Review of Biomedical Engineering, vol. 12, pp. 55-85, 2010.

[2] M. Luo, Y. Feng, T. Wang, and J. Guan, "Micro-/Nanorobots at Work in Active Drug Delivery," Advanced Functional Materials, vol. 28, no. 25, pp. 1-23, 2018.

[3] L. Kong, J. Guan, and M. Pumera, "Microand nanorobots based sensing and biosensing," Current Opinion in Electrochemistry, vol. 10, pp. 174-182, 2018.

[4] S. Heo, T. Wiguna, H. C. Park, and N. S. Goo, "Effect of an Artificial Caudal Fin on the Performance of a Biomimetic Fish Robot Propelled by Piezoelectric Actuators," Journal of Bionic Engineering, vol. 4, no. 3, pp. 151-158, 2007.

[5] B. Chen, S. Jiang, Y. Liu, P. Yang, and S. Chen, "Research on the Kinematic Properties of a Sperm-Like Swimming Micro Robot," Journal of Bionic Engineering, vol. 7, pp. S123-S129, 2010.

[6] X. Ma, A. Jannasch, U. R. Albrecht, K. Hahn, A. Miguel-López, E. Schäffer, and S. Sánchez, "Enzyme-Powered Hollow Mesoporous Janus Nanomotors," Nano Letters, vol. 15, no. 10, pp. 7043-7050, 2015.

[7] B. Jang, E. Gutman, N. Stucki, B. F. Seitz, P. D. Wendel-García, T. Newton, J. Pokki, O. Ergeneman, S. Pané, Y. Or, and B. J. Nelson, "Undulatory Locomotion of Magnetic Multilink Nanoswimmers," Nano Letters, vol. 15, no. 7, pp. 4829-4833, 2015.

[8] M. Xuan, Z. Wu, J. Shao, L. Dai, T. Si, and Q. He, "Near Infrared Light-Powered Janus Mesoporous Silica Nanoparticle Motors," 
Journal of the American Chemical Society, vol. 138, no. 20, pp. 6492-6497, 2016.

[9] Z. Wu, T. Li, J. Li, W. Gao, T. Xu, C. Christianson, W. Gao, M. Galarnyk, Q. He, L. Zhang, and J. Wang, "Turning erythrocytes into functional micromotors," ACS Nano, vol. 8, no. 12, pp. $12041-12048,2014$.

[10] K. Kim, X. Xu, J. Guo, and D. L. Fan, "Ultrahigh-speed rotating nanoelectromechanical system devices assembled from nanoscale building blocks," Nature Communications, vol. 5, pp. 1-9, 2014.

[11] B. Sun, G. Wood, and S. Miyashita, "Milestones for autonomous in vivo microrobots in medical applications," Surgery (United States), vol. 169, no. 4, pp. 755-758, 2021.

[12] E. Mancha, J. Traver, I. Tejado, J. Prieto, B. Vinagre, and V. Feliu, "Artificial flagellum microrobot. Design and simulation in COMSOL." Advances in Intelligent Systems and Computing, 2018, vol. 693.

[13] J. E. Traver, I. Tejado, C. Nuevo-Gallardo, M. A. López, and B. M. Vinagre, "Performance study of propulsion of N-link artificial Eukaryotic flagellum swimming microrobot within a fractional order approach: From simulations to hardware-in-the-loop experiments," European Journal of Control, vol. 58, pp. 340-356, 2021.

[14] G. Hwang, A. Mizushima, E. Lebrasseur, K. Misumi, N. Usami, A. Higo, and Y. Mita, "Mobile microrobotic cleaner in microfluidics," Sensors and Actuators A: Physical, vol. 318, p. 112502, 2021.

[15] I. S. Khalil, H. C. Dijkslag, L. Abelmann, and S. Misra, "MagnetoSperm: A microrobot that navigates using weak magnetic fields," $A p$ plied Physics Letters, vol. 104, no. 22, 2014.

[16] J. Donea, S. Giuliani, and J. P. Halleux, "An arbitrary lagrangian-eulerian finite element method for transient dynamic fluid-structure interactions," Computer Methods in Applied Mechanics and Engineering, vol. 33, no. 1-3, pp. 689-723, 1982.

[17] J. W. Thompson and W. J. O'Connor, "Wave-based attitude control of spacecraft with fuel sloshing dynamics," Proceedings of the ECCOMAS Thematic Conference on Multibody Dynamics 2015, Multibody Dynamics 2015, vol. LXIII, pp. 968-977, 2015.

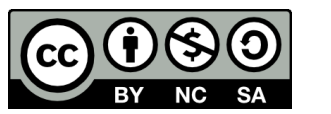

(C) 2021 by the authors. Submitted for possible open access publication under the terms and conditions of the Creative Commons Attribution CC BY-NC-SA 4.0 license (https://creativecommons.org/licenses/by-ncsa/4.0/deed.es). 